\title{
Epidemic Dynamics of COVID-19 Based on SEAIUHR Model Considering Asymptomatic Cases in Henan Province, China
}

\section{Chunyu Li}

Shandong University

Yuchen Zhu

Shandong University

Chang Qi

Shandong University

Lili Liu

Shandong University

Dandan Zhang

Shandong University

Xu Wang

Shandong University

Kaili She

Shandong University

Yan Jia

Shandong University

Tingxuan Liu

Shandong University

Momiao Xiong

University of Texas Health Science Center at San Antonio

Xiujun Li ( $\nabla$ xjli@sdu.edu.cn )

Shandong University https://orcid.org/0000-0001-7771-2725

\section{Research Article}

Keywords: COVID-19, asymptomatic cases, infectious dynamic model, the effective reproductive number, prevention and control measures

Posted Date: August 4th, 2020

DOl: https://doi.org/10.21203/rs.3.rs-50050/v1 
License: (c) (i) This work is licensed under a Creative Commons Attribution 4.0 International License. Read Full License 


\section{Epidemic dynamics of COVID-19 based on SEAIUHR model considering asymptomatic cases in Henan province, China}

Chunyu Li $i^{1}$, Yuchen Zhu ${ }^{1}$, Chang Qi ${ }^{1}$, Lili Liu ${ }^{1}$, Dandan Zhang ${ }^{1}$, Xu Wang ${ }^{1}$, Kaili She ${ }^{1}$, Yan $\mathrm{Jia}^{1}$, Tingxuan Liu ${ }^{1}$, Momiao Xiong ${ }^{2}$, Xiujun $\mathrm{Li}^{1}{ }^{*}$

${ }^{1}$ Department of Biostatistics, School of Public Health, Cheeloo College of Medicine, Shandong University, Jinan, Shandong, China

${ }^{2}$ Department of Biostatistics and Data Science, School of Public Health, University of Texas Health Science Center at Houston, Houston, TX, US.

${ }^{*}$ Corresponding author

E-mail: xjli@sdu.edu.cn 


\section{Abstract}

2 Background: New coronavirus disease (COVID-19), an infectious disease caused by

3 a type of novel coronavirus, has emerged in various countries since the end of 2019 and

4 caused a global pandemic. Many infected people went undetected because their

5 symptoms were mild or asymptomatic, but the proportion and infectivity of

6 asymptomatic infections remained unknown. Therefore, in this paper, we analyzed the

7 proportion and infectivity of asymptomatic cases, as we as the prevalence of COVID-

819 in Henan province.

9 Methods: We constructed SEAIUHR model based on COVID-19 cases reported from 21 January to 26 February 2020 in Henan province to estimate the proportion and

11 infectivity of asymptomatic cases, as we as the change of effective reproductive number, $R_{t}$. At the same time, we simulated the changes of cases in different scenarios by changing the time and intensity of the implementation of prevention and control measures.

Results: The proportion of asymptomatic cases among COVID-19 infected individuals was $42 \%$ and infectivity of asymptomatic cases was $10 \%$ of that symptomatic ones. The basic reproductive number $R_{0}=2.73$, and $R_{t}$ dropped below 1 on 1 February under a series of measures. If measures were taken five days earlier, the number of cases

19 would be reduced by $2 / 3$, and after 5 days the number would more than triple.

Conclusions: In Henan Province, the COVID-19 epidemic spread rapidly in the early stage, and there were a large number of asymptomatic infected individuals with relatively low infectivity. However, the epidemic was quickly brought under control 
with national measures, and the earlier measures were implemented, the better.

Keywords: COVID-19; asymptomatic cases; infectious dynamic model; the effective reproductive number; prevention and control measures;

\section{Background}

In early December 2019, the first case of pneumonia with unknown cause was reported. The disease was later confirmed to be coronavirus disease (COVID-19) caused by a type of novel coronavirus[1,2]. The rapid increase in confirmed cases and subsequent secondary outbreaks in many countries of the world had caused concern on an international scale. As a result, World Health Organization announced the COVID19 outbreak a Public Health Emergency of International Concern on 31 January and eventually classified it as a pandemic on 11 March[3]. As of 19 July 2020, 14 million COVID-19 cases and 597583 deaths have been confirmed globally, including 85937 confirmed cases in China[4]. Although the number of confirmed cases was staggering, it was only the sicker part of those infected. Li et al. used Metapopulation model to estimate that $86 \%$ of the infections before 23 January 2020 were undetected in Wuhan[5]; Chinazzi et al. used GLEAM model to estimate that only one out of four cases were detected and confirmed in Mainland China by 1 February 2020[6]; Hao et al. used SAPHIRE model to estimate that $87 \%$ of the infections before 8 March were unascertained in Wuhan[7]. And some even suggested that most infections were caused by undetected cases[5,8]. A significant proportion of these undetected infected 
individuals were asymptomatic[7], and although they had no symptoms, their viral load was comparable to that of the confirmed cases[9], making them somewhat infectious[10].

The fraction of asymptomatic cases is a critical epidemiological characteristic that modulates the pandemic potential of emergent respiratory virus, and an important parameter in estimating the disease burden and evaluating the effectiveness of prevention and control measures[5,11-13]. Estimates of the proportion of asymptomatic cases will improve the understanding of COVID-19 transmission and the spectrum of diseases it causes, thereby providing insight into the spread of epidemics[13]. But the estimated proportion of asymptomatic infected individuals varied widely from place to place. A recent analysis of 21 retrieved reports by the Centre for Evidence Based medicine in Oxford found that estimates of asymptomatic COVID-19 cases ranged from $5 \%$ to $80 \%[14]$. And current study only shows that asymptomatic infected individuals are less contagious than symptomatic ones, but there is no consensus on how contagious they are $[15,16]$. Therefore, it is important to estimate the proportion and infectivity of asymptomatic cases in various regions. Taking Henan province as an example, we used a model-inference framework to explore the proportion and infectivity of asymptomatic cases, so as to estimate the prevalence of COVID-19 and evaluate the effect of prevention and control measures.

\section{Methods}

\section{Study area}


The study area is located in east-central part of China $\left(31^{\circ} 23^{\prime}\right.$ to $36^{\circ} 22^{\prime}$ north latitude, $110^{\circ} 21^{\prime}$ to $116^{\circ} 39^{\prime}$ east longitude, Fig 1), with a population of more than 96 million and an area of $167,000 \mathrm{~km}^{2}$. Most of Henan is located in the warm temperature zone and has the characteristic of climate transition from plain to hills and mountains from east to west.

\section{Source of data}

All data were obtained from official websites of Provincial and Municipal Health Commissions (Additional file 1), which published COVID-19 case data and information. The case data included the number of newly confirmed cases, cured cases and deaths per day. The case information included age, gender, exposure history, date of symptom onset, and activity trajectory of confirmed cases. The identifiable personal information was removed for privacy protection.

\section{Case definition}

Although the definition of COVID-19 cases has been changed several times, which has greatly affected the observed epidemic curve in Wuhan[17], the change of cases in Henan province has been relatively stable, and the diagnosis of all cases in this study were based on the sixth edition of Diagnosis and Treatment Scheme for COVID-19 released by the National Health Commission of China[18]. A laboratory-confirmed case was defined if the patient had a positive test of SARS-CoV-2 virus by the real-time reverse-transcription-polymerase-chain-reaction (RT-PCR) assay or high-throughput sequencing of nasal and pharyngeal swab specimens. Only laboratory-confirmed cases were included in this study. 


\section{Modeling the epidemic of COVID-19 in Henan province}

To consider asymptomatic infected individuals, we constructed the susceptibleexposed-asymptomatic-confirmed-unconfirmed symptomatic-hospitalized-removed (SEAIUHR) model by extending the classic susceptible-exposed-infectious-removed (SEIR) model to include asymptomatic cases, unconfirmed symptomatic cases who did not seek medical attention or get tested for mild symptoms, and quarantined confirmed cases. In this model, we divided the population into seven compartments: S(susceptible), E(latent), A(asymptomatic infectious), I(confirmed symptomatic infectious), U(unconfirmed symptomatic individuals), $\mathrm{H}$ (hospitalized) and $\mathrm{R}$ (removed). Susceptible individuals could acquire the virus after contacting with infected cases (both symptomatic and asymptomatic), and became latent when they were infected but noninfectious. After a period of time, some of the latent individuals developed into symptomatic infections, some of whom were confirmed and treated until they progressed into the removed stage and some went unconfirmed because they did not have present themselves to healthcare facilities or to get tested for mild symptoms; others developed into asymptomatic infections and remained infectious until they progressed into the removed stage. Removed stage included individuals who were recovered or died.

Dynamic of these seven parts over time could be expressed by the following ordinary differential equation: 


$$
\left\{\begin{array}{l}
\frac{d S}{d t}=-\frac{\beta_{t} S(I+U)}{N}-\frac{\beta_{t} \theta S A}{N} \\
\frac{d E}{d t}=\frac{\beta_{t} S(I+U)}{N}+\frac{\beta_{t} \theta S A}{N}-\frac{E}{Z} \\
\frac{d I}{d t}=\frac{\left(1-\mu_{1}-\mu_{2}\right) E}{Z}-\frac{I}{r_{1}} \\
\frac{d A}{d t}=\frac{\mu_{1} E}{Z}-\frac{A}{r_{2}} \\
\frac{d U}{d t}=\frac{\mu_{2} E}{Z}-\frac{U}{r_{3}} \\
\frac{d H}{d t}=\frac{I}{r_{1}}-\frac{H}{r} \\
\frac{d R}{d t}=\frac{H}{r}+\frac{A}{r_{2}}+\frac{U}{r_{3}}
\end{array}\right.
$$

110 Where $\beta_{t}$ was the transmission rate due to symptomatic infected individuals at time $t$,

111 defined as the proportion of cases from susceptible individuals to infected individuals,

112 both asymptomatic and symptomatic, caused by symptomatic infected cases; $\theta$ was

113 the ratio of the transmission rate due to asymptomatic over symptomatic cases; $\mu_{1}$ and

$114 \mu_{2}$ were the proportion of the asymptomatic and unconfirmed symptomatic cases

115 among infected individuals, respectively; $z$ was the latent period; $r_{1}, r_{2}$ and $r_{3}$

116 were infectious periods of confirmed symptomatic, asymptomatic and unconfirmed

117 symptomatic cases, respectively and $r$ was the duration from hospitalization to

118 recovery or death. Assume that $N=S+E+I+A+U+H+R$.

119 The differential equations in the model were numerically solved using a $4^{\text {th }}$ order

120 Runge-Kutta (RK4) method. Specifically, for each step of the algorithm, each term on

121 the right side of equation was determined using a random sample of the Poisson

122 distribution[5].

123 On 25 January, Henan province implemented first-level public health emergency response to the epidemic and took a series of prevention and control measures, such as 
traffic restriction, quarantine and so on[19]. We assumed that these major government measures caused the transmission rate to change from a constant rate to a time dependent exponentially decreasing rate[20].

Then, the formula of $\beta_{t}$ could be expressed by the following step function:

$$
\beta_{t}=\left\{\begin{array}{l}
\beta_{0} \quad, t<=t_{1} \\
\beta_{0} * \exp \left(-a *\left(t-t_{1}\right)\right), t>t_{1}
\end{array}\right.
$$

Where $\beta_{0}$ was the transmission rate due to symptomatic infected individuals before implementing measures; $a$ was the decreasing rate of transmission rate; $t_{1}$ was the date to start implementing measures.

The effective reproductive number, $R_{t}$, could be computed as

$$
R_{t}=\left(1-\mu_{1}-\mu_{2}\right) \beta_{t} r_{1}+\mu_{1} \theta \beta_{t} r_{2}+\mu_{2} \beta_{t} r_{3}
$$

In the initial state, namely, $t=0, R_{t}$ was the basic reproductive number $\left(R_{0}\right)$.

\section{Estimation of parameters in the model}

Initial states and parameters setting in the model were presented in Table 1. We assumed that the initial latent population, asymptomatic infected population and unconfirmed symptomatic cases were drawn from uniform distribution $[0,10]$; the initial confirmed symptomatic infected population was 0 ; and the rest of Henan province were susceptible. For parameters, we estimated $\beta_{0}, \mu_{1}, \mu_{2}, \theta$ and $\alpha$ by assuming that the values of parameters $z, r_{1}, r_{2}, r_{3}$ and $r$ were fixed throughout the process. We assumed that the initial values of each parameter to be estimated were drawn using Latin hypercube sampling in uniform distribution. The initial ranges of $\mu_{1}$, $\mu_{2}$ and $\theta$ were chosen to cover most possible values, i.e. [0,1]; the initial range of $\alpha$ was selected to more broadly cover what the previous research covered[20]; The initial 
range of $\beta$ was selected from the widest possible range of basic reproductive number $\left(R_{0}\right)$;

We used the Ensemble Adjustment Kalman Filter (EAKF) to infer epidemiological parameters of the model based on the number of cases presenting symptoms per day in Henan province[21-23]. The EAKF is a data assimilation algorithm, which only needs hundreds of ensemble members to obtain good results, especially suitable for the estimation of high-dimensional parameters of the model[24,25], and has been successfully applied to epidemics such as cholera and influenza[22,25]. In this study, we used 1000 ensemble numbers and 1,000 independent realizations to infer parameters and their corresponding $95 \%$ confidence intervals (Cls).

\section{Sensitivity analysis}

Synthetic testing. Before applying the model-inference framework to the number of new symptomatic cases per day, we tested the effect of model-inference framework with model-generated outbreak data. Specifically, we fixed the parameters of the model and used the model to generate synthetic outbreak data. We then applied the EAKF algorithm to the synthetic daily outbreak data, and assessed the model-inference framework by analyzing whether the model could fit the synthetic outbreak data and estimate parameters.

\section{Sensitivity of parameters estimation to the range of initial states and values of fixed} parameters. In initial states, the quantities of $E_{0}, A_{0}$ and $U_{0}$ were unknown, and our assumptions may affect the estimation of other parameters. Therefore, this study simultaneously investigated the results of parameters estimation when shortening and 
expanding their ranges. At the same time, we changed values of fixed parameters respectively to test the robustness of our results.

\section{Results}

As shown in Fig 2, Our model could fit the observed data well and accurately capture the peak and tendency of the epidemic. The numbers of cases observed were within the confidence interval estimated by the model, except for a few days in the later stages of the outbreak.

The mean estimation of transmission rate due to symptomatic infected individuals was 1.14 (95\% CI:1.07-1.23) at the beginning of the epidemic and the decreasing rate of transmission rate after implementing prevention and control measures was $0.16(95 \%$ CI: 0.12-0.19). Our model estimated that the asymptomatic rate among COVID-19 infected individuals was $42 \%$ (95\% CI: $41-47 \%$ ), and the mean ratio of the transmission rate of asymptomatic over symptomatic cases was 0.1 (95\% CI: $0.02-0.11 \%)$. At the same time, our model estimated that $11 \%$ (95\% CI:0.09-0.22) of infected individuals were unconfirmed symptomatic cases who did not seek medical attention or get tested for mild symptoms (Table 2). Then, the fraction of undocumented infections in Henan province was 53\% (95\% CI:50\%-68\%). Based on above parameters, we estimated the average effective reproduction number, $R_{t}$, to be $2.73(95 \%$ CIs:2.64-3.31) at the beginning of the epidemic, which was equal to the basic reproduction number $\left(R_{0}\right)$. With the implementation of measures, $R_{t}$ fell below 1 on 1 Feburary. The first-level public health emergency response was implemented to COVID-19 
on 25 January in Henan. Assuming that epidemic parameters remained unchanged but the time of epidemic response was changed, epidemic dynamics estimated by the model were shown in Fig 3. By 26 February, implementing measures 2 days earlier could reduce infections by $22 \%$ and 5 days earlier by $63 \%$. When the implementation was delayed by 2 days, the number of infections increased by $78 \%$, and 5 days later by $219 \%$. Assuming that epidemic parameters and the implementation time remained unchanged but the intensity of the implementation of measures, namely the rate of reduction of the infection rate, was changed, epidemic dynamics estimated by the model were shown in Fig 4. By 26 February, 30\% reduction in implementation intensity would increase the number of infections by $80 \%$, and a $50 \%$ reduction would increase by $172 \%$. When the implementation intensity was increased to 1.5 times of the current level, the number of infections would reduce by $7 \%$ and doubling that would reduce by $20 \%$ (Table 3 ).

We used model-generated synthesis outbreak in free simulation to test the modelinference-framework. The results were shown in Fig 5 and Table 4. All generated values were within the confidence interval estimated by the model and values of all parameters were within the estimated $95 \%$ confidence interval, which demonstrated the ability of the model-inference-framework to fit the synthetic outbreak data and estimate all five target model parameters simultaneously and accurately.

Results of parameters estimation when changing the range of initial states and values of fixed parameters were shown in Additional file 2. It could be seen that values of all epidemiological parameters fallen around the estimated values, with small fluctuations, indicating that estimated results of our model were robust. 


\section{Discussion}

We constructed a SEAIUHR model to fit the number of COVID-19 cases in Henan province and conducted a detailed analysis of the epidemic dynamics. asymptomatic infections among all infections of the disease, is important for estimating the true burden of disease and its transmission potential. At present, results of different studies on the asymptomatic proportion vary greatly[14]. We estimated that the proportion of asymptomatic infections among infected individuals during the entire epidemic was $42 \%$ in Henan province, within the confidence interval of estimated asymptomatic rate of 13 cases imported from Wuhan to Japan[13]. But it was higher than that of Diamond Princess cruise ship, which showed that only $17.9 \%$ of those infected were asymptomatic[26]. It could be that passengers and crew on the diamond princess were not drawn from a random sample of the general population, most of whom were older than 60 years and tended to have more severe symptoms after infection. Due to the absence of clinical symptoms such as coughing and sneezing, the chance of transmission caused by the pathogen being discharged from the body of an asymptomatic case was relatively less than that of a symptomatic one. Our model

231 estimated that the mean ratio of transmission rate due to asymptomatic over symptomatic cases was 0.1 , corresponding to study showing that prolonged exposure to infected persons and short exposure to symptomatic persons (such as coughing) is associated with a higher risk of transmission, while short exposure to asymptomatic 
contacts is associated with a lower risk of transmission[27].

The fraction of undocumented infections, including asymptomatic cases and unconfirmed symptomatic cases who did not seek medical attention or get tested for mild symptoms, was lower than that of Wuhan in the early stage of the epidemic[5-7], which may caused by following reasons. Firstly, in the early stage, the medical configuration was not perfect and public awareness was still insufficient, while the undocumented rate gradually decreased with the development of the epidemic[5,9,28]; secondly, contact tracing measures implemented in China may become unfeasible when the number of cases in Wuhan rose sharply in the early stage[3]. Finally, we need to point out that the differences in the estimated proportions of asymptomatic cases and unconfirmed symptomatic cases may due to unidentifiability of parameters in epidemiological models. The theoretic analysis of identifiability of parameters in epidemiological models needs to be done in the future.

Basic reproductive number $\left(R_{0}\right)$ is an important parameter to determine whether an infectious disease is prevalent or not. If $R_{0}<1$, infectious disease would gradually decline and die out without epidemic; if $R_{0}>1$, an epidemic would break out. In this study, our estimate of $R_{t}=2.73$ at the beginning of the epidemic measured the basic reproductive number $R_{0}$, that is, without intervention, each infected individual could infect an average of 2.73 susceptible individuals. This result was similar to those studies in other regions of China[29-31]. Combined with the latent period, the number of cases without intervention would increase exponentially[32,33]. However, Henan province implemented first level response on 25 January and adopted a series of prevention and 
control measures. The isolation treatment of confirmed cases and the testing of suspected cases aimed at removing infected individuals from the process of transmission; the closing of public places and the change of crowd behavior were to protect susceptible groups; Contact tracing, which identified possible chains of transmission between known infected persons and their contacts to quarantine suspected cases at home before symptoms appear, affected both susceptible and asymptomatic individuals and can effectively interrupt transmission. It was under these measures that $R_{t}$ dropped below 1 on 31 January, indicating that national strong prevention and control measures have achieved remarkable results.

To explore the impact of timing and intensity of the implementation of measures on the epidemic, we estimated the changes of the cases under different implementation time and intensity. We found that measures were taken 5 days earlier would reduce the number of cases by $2 / 3$, while the number of cases would more than triple when delayed by 5 days, suggesting that the earlier measures were implemented, the better. Changes in intensity showed that reducing the intensity by half would increase the number of cases by $80 \%$, while doubling the intensity would reduce the number of cases by only $20 \%$. Reducing the intensity would cause large changes in cases, while increasing the intensity would not have significant effects, although increasing the intensity would consume a lot of manpower and material resources, which indicated that the current intensity was properly handled.

This study also has some limitations. Firstly, our estimate of the asymptomatic proportion was obtained by model, which could not be generalized because it has not 
been confirmed by serological investigation. Secondly, this study estimated the average asymptomatic infection rate in Henan province over time, but the asymptomatic rate may vary in different periods of the epidemic.

\section{Conclusions}

We found that the epidemic situation developed rapidly in Henan province, and there were a large number of asymptomatic infected individuals with relatively low infectivity. However, with national strong prevention and control measures, the epidemic was quickly brought under control, and we estimated that the earlier the measures were implemented, the better. This study could provide a reference for the prevention and control of possible secondary outbreaks of COVID-19.

\section{Abbreviations}

COVID-19: coronavirus disease 2019

$R_{0}$ : the basic reproductive number

$R_{t}:$ the effective reproductive number

SEIAUHR model: susceptible-exposed-asymptomatic-confirmed-unconfirmed symptomatic-hospitalized-removed model

\section{Declarations}

\section{Ethics approval and consent to participate}

Not applicable. 
Not applicable.

\section{Availability of data and materials}

304 The dataset used in the study are available from the corresponding author on reasonable 305 request.

306 Competing interests

307 The authors declare that they have no competing interests.

\section{$308 \quad$ Funding}

309 This research was supported by the National Natural Science Foundation of China 310 (Grant No. 81673238) and COVID-19 Emergency Research Project of Shandong

311 University (Grant No. 2020XGC01) and National Key Research and Development

312 Program of China (Grant No. 2019YFC1200500, 2019YFC1200502). Founders have 313 no role in the design of the study and collection, analysis, interpretation of data or in 314 writing the manuscript.

\section{Authors' contributions}

316 CYL and XJL conceived of and designed the research. CHL, YCZ, CQ, LLL, DDZ, 317 XW, KLS, YJ and TXL did the analyses. CYL wrote and revised the paper. MMX and 318 XJL contributed to the writing and revisions. All the authors have read and approved 319 the submitted version. All the authors have agreed both to be personally accountable 320 for the author's own contributions and to ensure that questions related to the accuracy 321 or integrity of any part of the work .

\section{Acknowledgements}


We appreciate Health Commission of Henan Province and its subsidiaries for providing data for our research.

\section{Reference}

1. Chen N, Zhou M, Dong X, Qu J, Gong F, Han Y, et al. Epidemiological and clinical characteristics of 99 cases of 2019 novel coronavirus pneumonia in Wuhan, China: a descriptive study. Lancet. 2020;395(10223):507-13.

2. Guan W, Ni Z, Hu Y, Liang W, Ou C, He J, et al. Clinical Characteristics of Coronavirus Disease 2019 in China. N Engl J Med. 2020; 382(18):1708-1720.

3. Maier BF, Brockmann D. Effective containment explains subexponential growth in recent confirmed COVID-19 cases in China. Science. 2020;368(6492): 742-6.

4. WHO. Coronavirus disease (COVID-19) Situation Report-181. https://www.who.int/docs/default-source/coronaviruse/situation-reports/20200719covid-19-sitrep-181.pdf?sfvrsn=82352496_2. Accessed 20 Jul 2020.

5. Li R, Pei S, Chen B, Song Y, Zhang T, Yang W, et al. Substantial undocumented infection facilitates the rapid dissemination of novel coronavirus (SARS-CoV-2). Science. 2020;368(6490):489-93.

6. Chinazzi M, Davis JT, Ajelli M, Gioannini C, Litvinova M, Merler S, et al. The effect of travel restrictions on the spread of the 2019 novel coronavirus (COVID-19) outbreak. Science. 2020;368(6489):395-400.

7. Hao X, Cheng S, Wu D, Wu T, Lin X, Wang C. Reconstruction of the full transmission dynamics of COVID-19 in Wuhan. Nature. 2020; DOI: 
8. Qiu J. Covert coronavirus infections could be seeding new outbreaks. Nature. 2020; DOI: 10.1038/d41586-020-00822-x.

9. Zou L, Ruan F, Huang M, Liang L, Huang H, Hong Z, et al. SARS-CoV-2 viral load in upper respiratory specimens of infected patients. N. Engl. J. Med. 2020;382(12):1177-9.

10. Bai Y, Yao L, Wei T, Tian F, Jin DY, Chen L, et al. Presumed Asymptomatic Carrier Transmission of COVID-19. JAMA. 2020;323(14):1406-7.

11. Munster VJ, Koopmans M, van Doremalen N, van Riel D, de Wit E. A novel

12. Chan JFW, Yuan S, Kok KH, To KKW, Chu H, Yang J, et al. A familial cluster of pneumonia associated with the 2019 novel coronavirus indicating person-to-person transmission: a study of a family cluster. Lancet. 2020;395(10223):514-23.

13. Nishiura H, Kobayashi T, Miyama T, Suzuki A, Jung S mok, Hayashi K, et al. Estimation of the asymptomatic ratio of novel coronavirus infections (COVID-19). Int. J. Infect. Dis. 2020; 94:154-5.

14. Heneghan C, Brassey J, Jefferson T. COVID-19:What proportion are asymptomatic? Centre for Evidence-Based Medicine. 2020. https://www.cebm.net/covid-19/covid19-what-proportion-are-asymptomatic. Accessed 20 Jul 2020.

Outbreak in Call Center, South Korea. Emerg Infect Dis. 2020; 26(8):1666-70. 
16. Cheng HY, Jian SW, Liu DP, Ng TC, Huang WT, Lin HH. Contact Tracing Assessment of COVID-19 Transmission Dynamics in Taiwan and Risk at Different Exposure Periods before and after Symptom Onset. JAMA Intern Med. 2020; DOI: 10.1001/jamainternmed.2020.2020.

17. Tsang TK, Wu P, Lin Y, Lau EHY, Leung GM, Cowling BJ. Effect of changing case definitions for COVID-19 on the epidemic curve and transmission parameters in mainland China: a modelling study. Lancet Public Heal. 2020;5(5):e289-96.

18. National Health Commission of the Peoples's Republic of China. Clinical diagnosis and treatment guidance of 2019 novel coronavirus (COVID-19) caused pneumonia.http://www.nhc.gov.cn/yzygj/s7652m/202002/54e1ad5c2aac45c19eb54 1799bf637e9.shtml. Accessed 20 Jul 2020.

19. Zhang J, Litvinova M, Wang W, Wang Y, Deng X, Chen X, et al. Evolving epidemiology and transmission dynamics of coronavirus disease 2019 outside Hubei province, China: a descriptive and modelling study. Lancet Infect Dis. 2020;20(7):793-802.

20. Liu Z, Magal P, Seydi O, Webb G. Predicting the Cumulative Number of Cases for the COVID-19 Epidemic in China from Early Data. Math Biosci Eng. $2020 ; 17(4): 3040-51$.

21. King AA, Ionides EL, Pascual M, Bouma MJ. Inapparent infections and cholera dynamics. Nature. 2008;454(7206):877-80.

22. Pei S, Morone F, Liljeros F, Makse H, Shaman JL. Inference and control of the nosocomial transmission of methicillin-resistant Staphylococcus aureus. Elife. 
23. Anderson JL. An Ensemble Adjustment Kalman Filter for Data Assimilation. Mon 391 Weather Rev. 2001;129(12):2884-903.

24. Pei S, Kandula S, Yang W, Shaman J. Forecasting the spatial transmission of influenza in the United States. Proc Natl Acad Sci U S A . 2018;115(11):2752-7.

25. Portet S. A primer on model selection using the Akaike Information Criterion. Infect Dis Model. 2020;5:111-28.

26. Mizumoto K, Kagaya K, Zarebski A, Chowell G. Estimating the asymptomatic proportion of coronavirus disease 2019 (COVID-19) cases on board the Diamond Princess cruise ship, Yokohama, Japan, 2020. Eurosurveillance. 2020; 25(10):2000180.

27. Wiersinga WJ, Rhodes A, Cheng AC, Peacock SJ, Prescott HC. Pathophysiology, Transmission, Diagnosis, and Treatment of Coronavirus Disease 2019 (COVID-19): A Review. JAMA. 2020; DOI: 10.1001/jama.2020.12839.

28. Zhao S, Musa SS, Lin Q, Ran J, Yang G, Wang W, et al. Estimating the Unreported Number of Novel Coronavirus (2019-nCoV) Cases in China in the First Half of January 2020: A Data-Driven Modelling Analysis of the Early Outbreak. J Clin Med. $2020 ; 9(2): 388$

29. Liu Y, Gayle AA, Wilder-Smith A, Rocklöv J. The reproductive number of COVID19 is higher compared to SARS coronavirus. J Travel Med. 2020;27(2):1-4.

30. Li Q, Guan X, Wu P, Wang X, Zhou L, Tong Y, et al. Early transmission dynamics in Wuhan, China, of novel coronavirus-infected pneumonia. N. Engl. J. Med. 
31. Xu XW, Wu XX, Jiang XG, Xu KJ, Ying LJ, Ma CL, et al. Clinical findings in a group of patients infected with the 2019 novel coronavirus (SARS-Cov-2) outside of Wuhan, China: Retrospective case series. BMJ. 2020;368:m606.

32. Linton NM, Kobayashi T, Yang Y, Hayashi K, Akhmetzhanov AR, Jung S-M, et

al. Incubation Period and Other Epidemiological Characteristics of 2019 Novel Coronavirus Infections with Right Truncation: A Statistical Analysis of Publicly Available Case Data. J Clin Med. 2020;9(2):538.

33. Lauer SA, Grantz KH, Bi Q, Jones FK, Zheng Q, Meredith HR, et al. The incubation period of coronavirus disease 2019 (CoVID-19) from publicly reported confirmed cases: Estimation and application. Ann Intern Med. 2020;172(9):577-82.

34. He X, Lau EHY, Wu P, Deng X, Wang J, Hao X, et al. Temporal dynamics in viral shedding and transmissibility of COVID-19. Nat Med. 2020;26(5):672-5.

35. Wei WE, Li Z, Chiew CJ, Yong SE, Toh MP, Lee VJ. Presymptomatic Transmission of SARS-CoV-2-Singapore. Morb Mortal Wkly Rep. 2020;69(14):411-5.

36. Wang D, Hu B, Hu C, Zhu F, Liu X, Zhang J, et al. Clinical Characteristics of 138 Hospitalized Patients with 2019 Novel Coronavirus-Infected Pneumonia in Wuhan, China. JAMA. 2020;323(11):1061-9. 
The asterisk represents the number of cases with symptoms observed on a daily basis; the curve shows the change in the average number of confirmed symptomatic cases per day estimated by the model; the light blue shade was the $95 \%$ confidence interval of the estimation.

Fig 3. Changes in the number of confirmed symptomatic cases when changing the

This figure showed Changes in the number of new confirmed symptomatic cases when changing the time of the implementation of measures. We could see that the earlier the measures were implemented, the better. increasing the intensity.

Fig 5. Comparison of the number of cases estimated and generated

453 The asterisk represents the number of cases with symptoms observed on a daily basis; the curve shows the change in the average number of confirmed symptomatic cases per 

the estimation.

Table 1. Initial states and parameters setting in the model of the main analysis

States or parameters

\section{States}

Susceptible $\left(S_{0}\right)$

Latent $\left(E_{0}\right)$

Confirmed symptomatic infectious $\left(I_{0}\right)$

Asymptomatic infectious $\left(A_{0}\right)$

Unconfirmed symptomatic infectious $\left(U_{0}\right)$

\section{Parameters}

latent $\operatorname{period}(z)$

Infectious period of confirmed symptomatic cases $\left(r_{1}\right)$

Infectious period of asymptomatic cases $\quad\left(r_{2}\right)$

Infectious period of unconfirmed symptomatic cases $\quad\left(r_{3}\right)$

Duration removed from hospitalization $(r)$

Transmission rate due to symptomatic cases $\left(\beta_{0}\right)$

Asymptomatic rate $\left(\mu_{1}\right)$

Undetected rate $\left(\mu_{2}\right)$

The ratio of transmission rate $(\theta)$

The decreasing rate of transmission rate $(\alpha)$ values or prior distribution

$$
96050000-E_{0}-I_{0}-A_{0}-U_{0}
$$$$
\mathrm{U}(0,10)
$$

0

$$
\mathrm{U}(0,10)
$$

$\mathrm{U}(0,10)$

3 days (Fixed)[2,27,33-35]

3.5 days (Fixed)[5,19,31,32]

5 days (Fixed)[16]

5 days (Fixed)[16]

10 days (Fixed)[36]

$\mathrm{U}(0.8,1.5)$ (Estimated)

$\mathrm{U}(0.02,1)$ (Estimated)

$\mathrm{U}(0.02,1)$ (Estimated)

$\mathrm{U}(0.02,1)$ (Estimated)

$\mathrm{U}(0.02,0.3)$ (Estimated) 
460 Table 2. Posterior estimates of key epidemiological parameters

\begin{tabular}{ll}
\hline Parameter & Mean $(95 \% \mathrm{CI})$ \\
\hline Transmission rate due to symptomatic cases $\left(\beta_{0}\right)$ & $1.14(1.07,1.23)$ \\
Asymptomatic rate $\left(\mu_{1}\right)$ & $0.42(0.41,0.47)$ \\
Undetected rate $\left(\mu_{2}\right)$ & $0.11(0.09,0.22)$ \\
The ratio of transmission rate $(\theta)$ & $0.10(0.02,0.11)$ \\
The decreasing rate of transmission rate $(\alpha)$ & $0.16(0.12,0.19)$ \\
\hline
\end{tabular}

This table showed posterior estimates of key epidemiological parameters using proposed model-inference framework. In the second column, estimated mean and 95\% confidence interval were outside and inside parentheses, respectively.

Table 3. Changes in the number of total cases when changing the time

or intensity of the implementation of measures

\begin{tabular}{cccc}
\hline Time & Intensity $^{\mathrm{b}}$ & Total cases & Percentage $^{\mathrm{c}}$ \\
\hline 23 January & $\alpha$ & $970(845,1010)$ & $78 \%$ \\
20 January & $\alpha$ & $458(388,471)$ & $37 \%$ \\
25 January & $\alpha$ & $1242(1162,1499)$ & $100 \%$ \\
27 January & $\alpha$ & $2205(1823,2226)$ & $178 \%$ \\
30 January & $\alpha$ & $3966(3092,3984)$ & $319 \%$ \\
25 January & $\alpha * 0.7$ & $2234(1818,2262)$ & $180 \%$ \\
25 January & $\alpha * 0.5$ & $3382(2595,3398)$ & $272 \%$ \\
\hline
\end{tabular}




\begin{tabular}{lccc}
\hline 25 January & $\alpha * 1.5$ & $1158(1021,1188)$ & $93 \%$ \\
25 January & $\alpha * 2.0$ & $988(886,1014)$ & $80 \%$ \\
\hline
\end{tabular}

${ }^{\mathrm{b}}$ The real strength is $\alpha$.

${ }^{c}$ As of 26 February, the percentage of the number of total confirmed symptomatic cases estimated by the model relative to the actual number of confirmed symptomatic cases at the specified time and intensity of implementation.

\section{Table 4. Results of Synthetic testing}

\begin{tabular}{cll}
\hline Parameters & True values & Estimated values $^{\mathrm{a}}$ \\
\hline$\beta$ & 1.1 & $1.14(1.06,1.22)$ \\
$\mu_{1}$ & 0.4 & $0.34(0.32,0.45)$ \\
$\mu_{2}$ & 0.1 & $0.09(0.07,0.23)$ \\
$\theta$ & 0.2 & $0.18(0.14,0.22)$ \\
$\alpha$ & 0.15 & $0.15(0.12,0.19)$ \\
\hline
\end{tabular}

${ }^{\text {a }}$ The estimated mean and 95\% confidence interval were outside and inside the parentheses.

\section{Additional file 1. Source of data}

\begin{tabular}{ll}
\hline Area & Website \\
\hline Henan Province & http://wsjkw.henan.gov.cn/ \\
Zhengzhou Shi & http://wjw.zhengzhou.gov.cn/ \\
Kaifeng Shi & http://www.kfwsjsw.gov.cn/ \\
\hline
\end{tabular}




\begin{tabular}{|c|c|}
\hline Luoyang Shi & http://www.lyws.gov.cn/ \\
\hline Pingdingshan Shi & http://www.pdswsjsw.gov.cn/ \\
\hline Anyang Shi & http://aywjw.anyang.gov.cn/ \\
\hline Hebi Shi & https://wsjkw.hebi.gov.cn/ \\
\hline Xinxiang Shi & http://www.xxswjw.gov.cn/ \\
\hline Jiaozuo Shi & http://www.jzswjw.gov.cn/ \\
\hline Puyang Shi & http://www.pyswjw.gov.cn/ \\
\hline Xuchang Shi & http://xcswjw.xuchang.gov.cn/ \\
\hline Luohe Shi & http://www.lhswjw.gov.cn/ \\
\hline Sanmenxia Shi & http://wjw.smx.gov.cn/ \\
\hline Nanyang Shi & http://nyws.nanyang.gov.cn/ \\
\hline Shangqiu Shi & http://www.sqwsjd.cn/ \\
\hline Xinyang Shi & http://www.hnxywjw.gov.cn/ \\
\hline Jiyuan Shi & http://www.zkwjw.gov.cn/ \\
\hline Zhumadian Shi & http://www.zmdwsj.gov.cn/ \\
\hline Jiyuan Shi & http://wjw.jiyuan.gov.cn/ \\
\hline
\end{tabular}

478 Additional file 2. Results of parameters estimation when values of initial states or

479 fixed parameters changed

\begin{tabular}{cccccc}
\hline & $\beta_{0}$ & $\mu_{1}$ & $\mu_{2}$ & $\theta$ & $\alpha$ \\
\hline $\mathrm{C} 1^{\mathrm{a}}$ & $1.14(1.05,1.22)$ & $0.44(0.42,0.47)$ & $0.09(0.07,0.21)$ & $0.08(0.02,0.09)$ & $0.15(0.12,0.18)$ \\
& & & & & \\
$\mathrm{C}^{\mathrm{b}}$ & $1.13(1.06,1.22)$ & $0.43(0.42,0.47)$ & $0.16(0.15,0.4)$ & $0.09(0.02,0.10)$ & $0.16(0.12,0.19)$
\end{tabular}




$\begin{array}{lllllll}\mathrm{C}^{\mathrm{c}} & 1.14(1.06,1.22) & 0.43(0.43,0.47) & 0.16(0.15,0.39) & 0.09(0.02,0.10) & 0.16(0.12,0.19) \\ Z=2^{\mathrm{d}} & 1.14(1.07,1.23) & 0.45(0.44,0.47) & 0.15(0.10,0.21) & 0.08(0.02,0.09) & 0.15(0.12,0.18) \\ Z=4^{\mathrm{e}} & 1.15(1.07,1.23) & 0.39(0.38,0.46) & 0.07(0.05,0.25) & 0.09(0.03,0.11) & 0.15(0.12,0.19) \\ r_{1}=2^{\mathrm{f}} & 1.14(1.06,1.22) & 0.37(0.36,0.46) & 0.12(0.10,0.29) & 0.14(0.08,0.16) & 0.15(0.12,0.18) \\ r_{1}=3^{\mathrm{g}} & 1.13(1.06,1.22) & 0.42(0.41,0.47) & 0.15(0.14,0.33) & 0.10(0.05,0.12) & 0.16(0.13,0.19) \\ r_{1}=4^{\mathrm{h}} & 1.14(1.06,1.22) & 0.44(0.43,0.47) & 0.12(0.10,0.28) & 0.08(0.02,0.08) & 0.16(0.12,0.19) \\ r_{2}=r_{3}=4^{\mathrm{i}} & 1.14(1.06,1.22) & 0.37(0.35,0.46) & 0.16(0.14,0.32) & 0.18(0.14,0.22) & 0.15(0.12,0.19) \\ r_{2}=r_{3}=6^{\mathrm{j}} & 1.14(1.07,1.23) & 0.40(0.39,0.47) & 0.17(0.15,0.34) & 0.12(0.07,0.15) & 0.16(0.12,0.19) \\ r_{2}=r_{3}=7^{\mathrm{k}} & 1.14(1.06,1.22) & 0.40(0.39,0.47) & 0.14(0.13,0.37) & 0.11(0.03,0.12) & 0.16(0.12,0.19)\end{array}$

480 The table showed the estimated results of each parameter when the initial range of 481 certain states or fixed parameters changed, where the estimated mean value was outside 482 the parenthesis and the $95 \%$ confidence interval was inside the parenthesis.

483 a change the initial range of $E_{0}, I_{0}$, and $U_{0}$ to $[0,5]$.

484 b change the initial range of $E_{0}, I_{0}$, and $U_{0}$ to $[0,15]$.

485 change the initial range of $E_{0}, I_{0}$, and $U_{0}$ to $[0,20]$.

$486 \quad{ }^{\mathrm{d}}$ Change the value of $z$ to $z=2$.

$487 \quad{ }^{\text {e}}$ Change the value of $z$ to $z=4$.

488 f Change the value of $r_{1}$ to $r_{1}=2$.

$489{ }^{\mathrm{g}}$ Change the value of $r_{1}$ to $r_{1}=3$.

490 h Change the value of $r_{1}$ to $r_{1}=4$.

491 i Change the value of $r_{2}$ and $r_{3}$ to $r_{2}=r_{3}=4$.

492 jंChange the value of $r_{2}$ and $r_{3}$ to $r_{2}=r_{3}=6$. 
${ }^{\mathrm{k}}$ Change the value of $r_{2}$ and $r_{3}$ to $r_{2}=r_{3}=7$. 


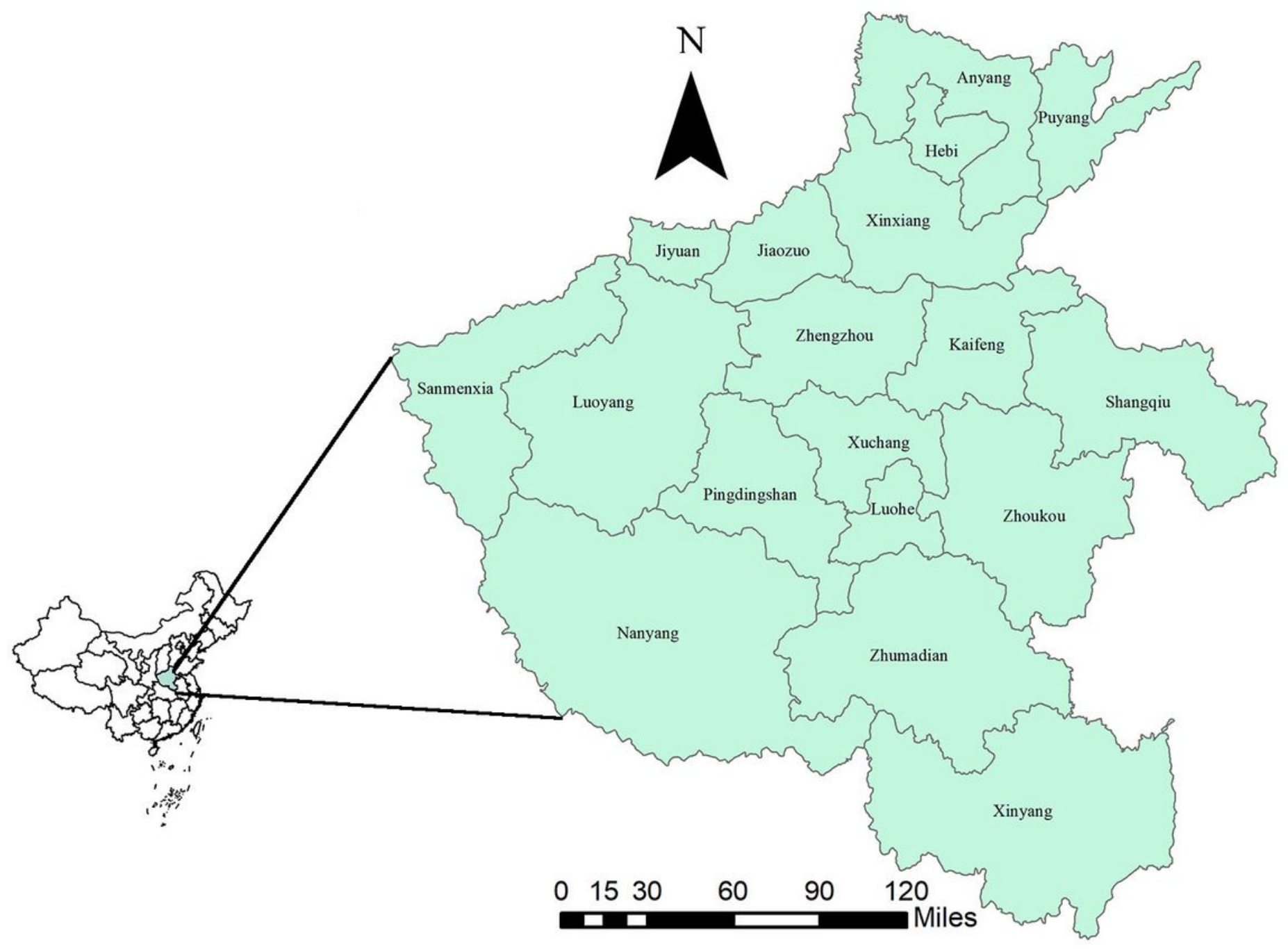

Figure 1

Location of this study. Note: The designations employed and the presentation of the material on this map do not imply the expression of any opinion whatsoever on the part of Research Square concerning the legal status of any country, territory, city or area or of its authorities, or concerning the delimitation of its frontiers or boundaries. This map has been provided by the authors. 


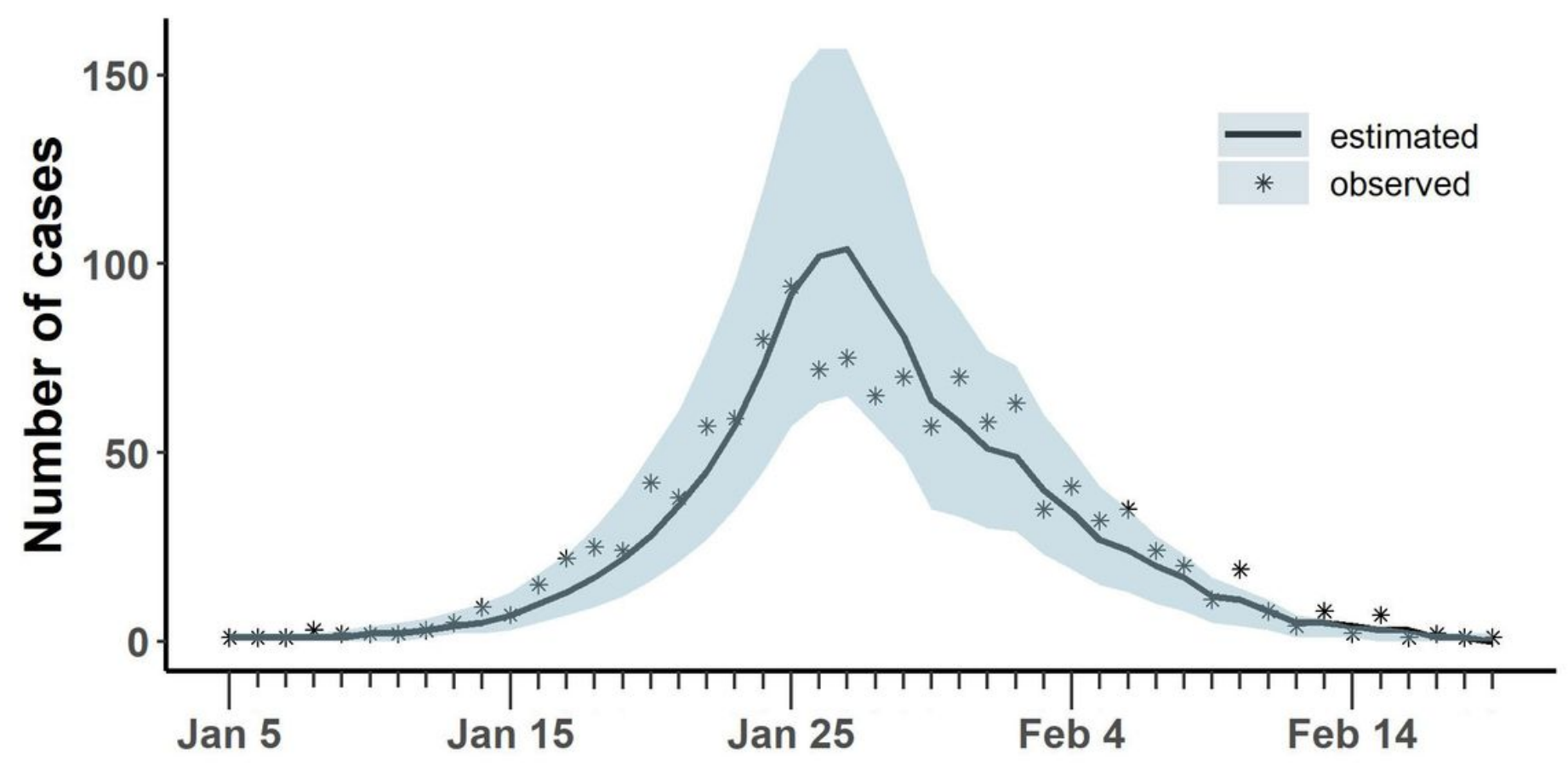

Figure 2

Comparison of the number of cases estimated and observed The asterisk represents the number of cases with symptoms observed on a daily basis; the curve shows the change in the average number of confirmed symptomatic cases per day estimated by the model; the light blue shade was the $95 \%$ confidence interval of the estimation.

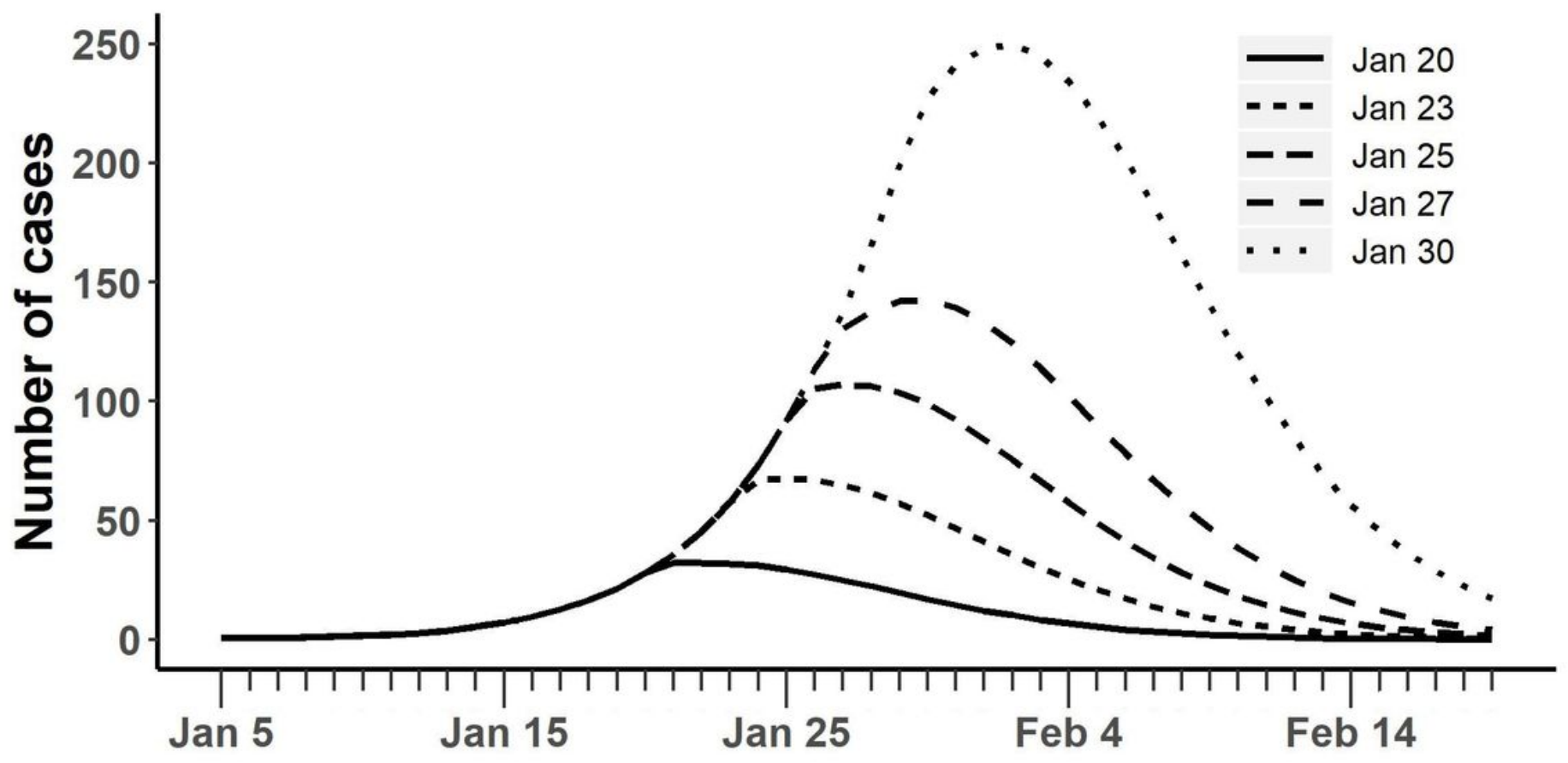

Figure 3 
Changes in the number of confirmed symptomatic cases when changing the time of the implementation This figure showed Changes in the number of new confirmed symptomatic cases when changing the time of the implementation of measures. We could see that the earlier the measures were implemented, the better.

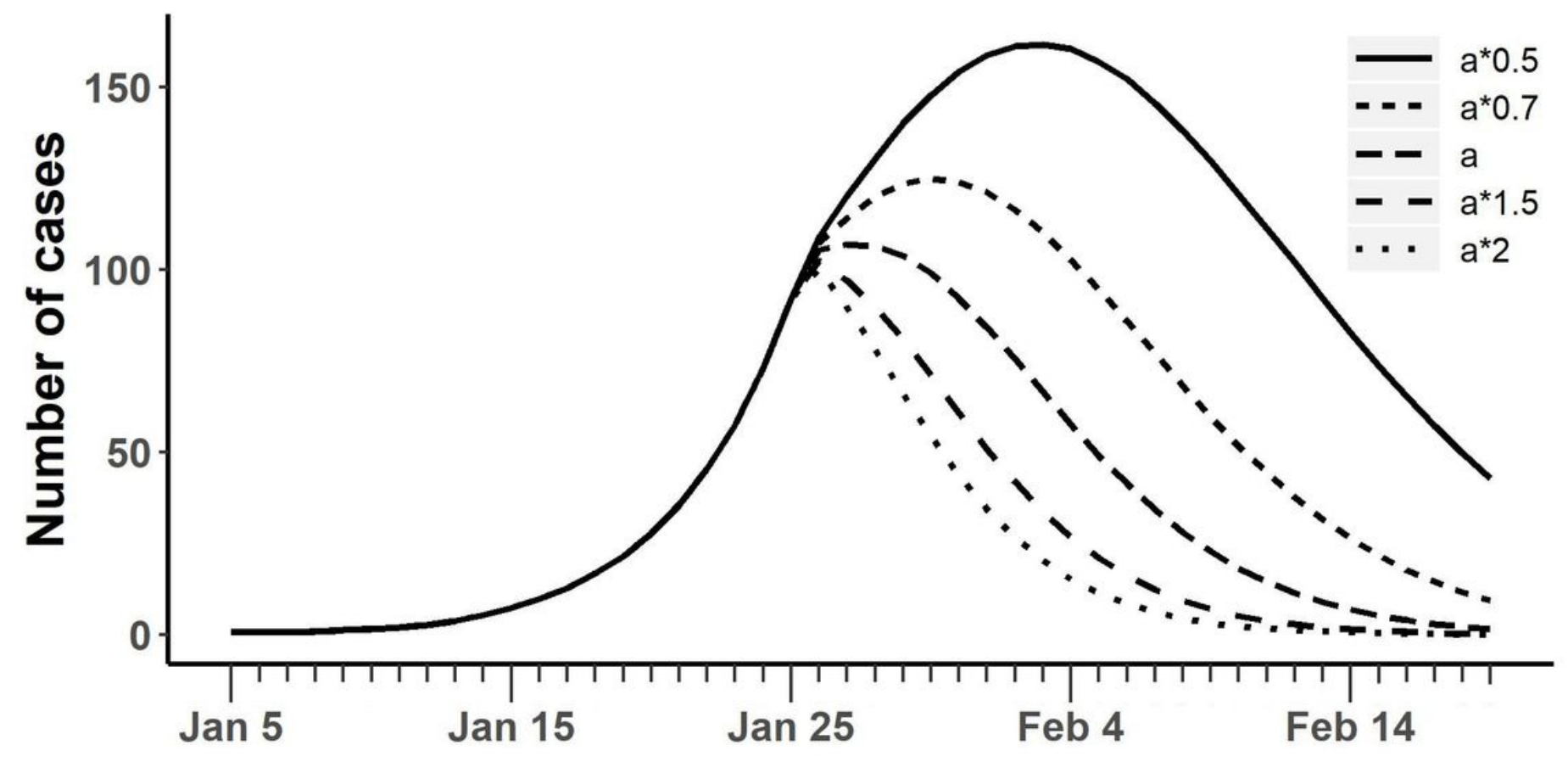

Figure 4

Changes in the number of confirmed symptomatic cases when changing the intensity of the implementation This figure showed Changes in the number of new confirmed symptomatic cases when changing the intensity of the implementation of measures. It could be seen that reducing the intensity would make the case change greatly, while the effect was not obvious when increasing the intensity. 


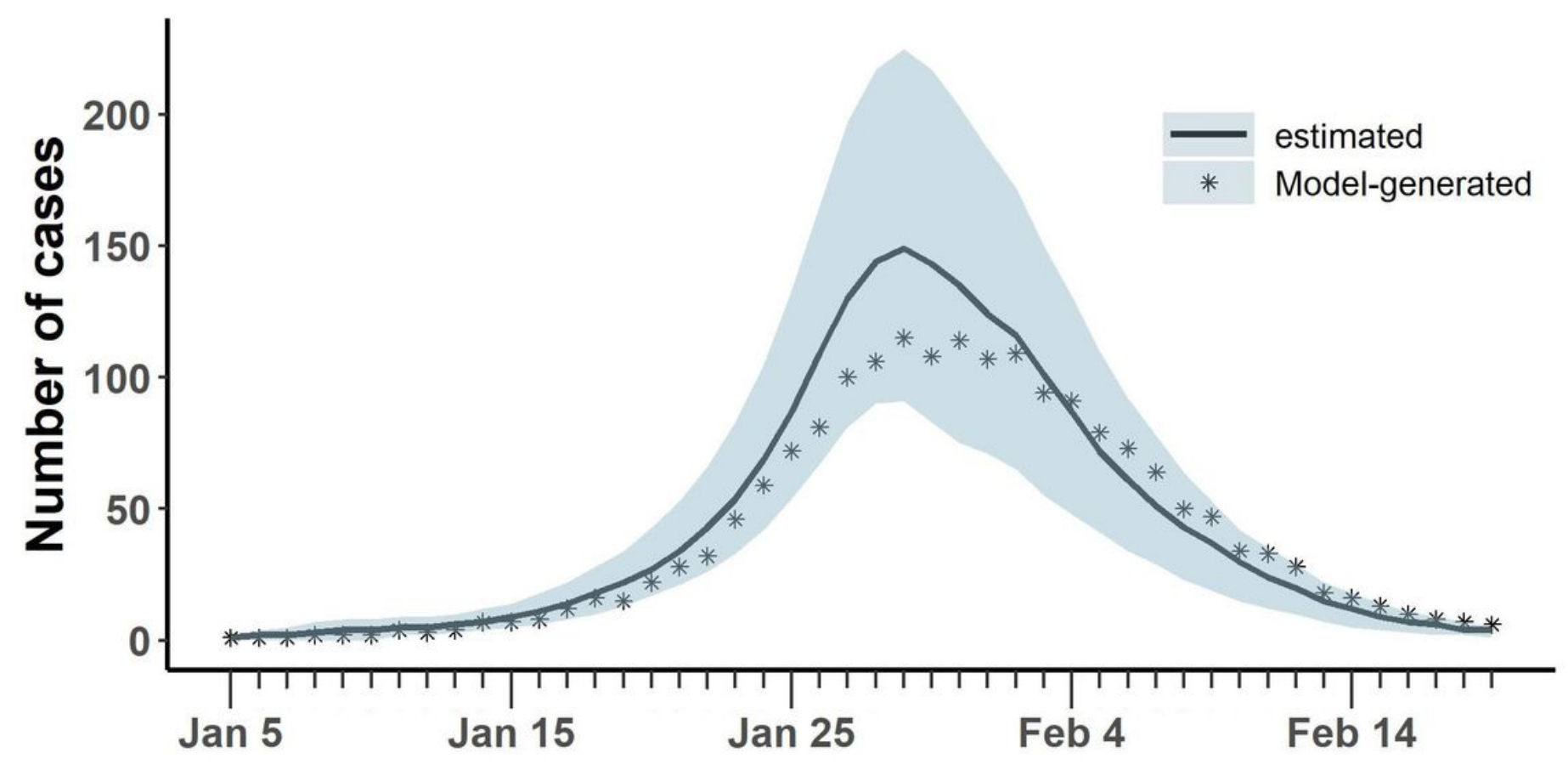

Figure 5

Comparison of the number of cases estimated and generated The asterisk represents the number of cases with symptoms observed on a daily basis; the curve shows the change in the average number of confirmed symptomatic cases per day estimated by the model; the light blue shade was the $95 \%$ confidence interval of the estimation. 